\author{
Journal of Research of the National Bureau of Standards
Volume 90, Number 2, March-April 1985
}

\title{
An Apparatus for Direct Fugacity Measurements on Mixtures Containing Hydrogen
}

\author{
Thomas J. Bruno \\ National Bureau of Standards, Boulder, CO 80303
}

\begin{abstract}
Accepted: January 7, 1985
An apparatus has been designed and constructed to allow measurements of fugacities in gaseous mixtures containing hydrogen. The apparatus makes use of a semipermeable membrane to allow a direct measurement of the partial pressure of a permeating component (in this case, hydrogen) in a mixture with a nonpermeating component. In this study, measurements were made on mixtures of hydrogen/methane and hydrogen/propane.

The apparatus is designed to operate at moderate and high temperatures (ambient to $250^{\circ} \mathrm{C}$ ) and moderate and high mixture pressures $(3$ to $50 \mathrm{MPa}$ ). The actual pressure range that is experimentally accessible is dependent on the fluid mixture under study, due to its influence on the pressure gradient across the semipermeable membrane.

The pressure measurements are done isothermally on a series of concentrations of the binary. Each mixture is characterized using a developmental gas-chromatograph which has been built especially for this work. The gas-chromatograph was calibrated using standard mixtures of hydrogen/methane and hydrogen/propane, prepared gravimetrically in our laboratory.

Using measured values of the mixture pressure, hydrogen partial pressure and mixture mole fraction at a given temperature, fugacity coefficients were determined using the virial equation. The measured values are compared with some previous data and general trends are discussed.
\end{abstract}

Key words: fugacity coefficients; fugacity measurements; gas-chromatograph; gas mixtures; hydrogen/methane; hydrogen/propane.

\section{Introduction}

The concept of fugacity owes its origin to Lewis $[1,2]^{1}$, who desired some measure of "escaping tendency" upon which to base his idea of equilibrium. His original use of partial molar free energy suffered from a negative infinity in the case of a gas in the limit of low pressure [3]. The concept of fugacity, which was devised to remedy this difficulty, is a convenient way to express the chemical potential of a substance in experimentally accessible terms. Since defining equations and useful functional relationships are presented in a variety

\begin{abstract}
About the Author, Paper: Thomas J. Bruno is a physical chemist in the Chemical Engineering Science Division of NBS' National Engineering Laboratory. The work described was supported financially by the Gas Research Institute.
\end{abstract}

\footnotetext{
${ }^{\mathrm{I}}$ Numbers in brackets refer to literature references
}

of sources [4-6], a discussion of the basics of fugacity will not be repeated here.

Fugacity is a most important quantity in experimental thermodynamics because of its theoretical significance and general usefulness. The phase equality of temperature, pressure, and component fugacity is the criterion for physical equilibrium of a system. In a system involving chemical reactions, fugacities appear in the equilibrium constants describing the multicomponent system. Departures from equilibrium can be described in terms of fugacity gradients [7]. Since fugacity is a measure of nonideality of a gaseous system, measurements of fugacity and fugacity coefficients are of value in research on equations of state, especially work involving mixtures.

There are several techniques by which fugacities in gas mixtures may be measured experimentally. The most generally used method involves consideration of the P-V-T surface of gas mixtures of composition $y_{i}$. The component fugacities $f_{\mathrm{i}}$ are then obtained from $[8,9]$ : 


$$
\ln f_{\mathrm{i}}=\ln P x_{\mathrm{i}}+\int\left(V_{\mathrm{i}} / R T-1 / P\right) \mathrm{d} P
$$

where $V_{\mathrm{i}}$ is a partial molar volume, and $x_{\mathrm{i}}$ is the mole fraction of component $\mathrm{i}$, and $P, T$ and $R$ have their usual meanings. The P-V-T-x data used in this type of measurement must be very precise, because of the loss of precision of derived thermodynamic functions. In addition, large quantities of PVT data are necessary for the successful exploitation of the above equation. One can also use the above equation with various equations of state as a predictive tool for fugacity.

There are more accurate techniques for experimental determination of fugacities in gas mixtures [10]; however these are applicable only in specific instances. One such case involves mixtures containing hydrogen. For these mixtures, a physical equilibrium technique using a semipermeable membrane is applicable. The principle of this technique is quite simple and is illustrated in figure 1. An experimental chamber is divided into two regions by a membrane permeable only to component $i$. The fugacity of pure component i at partial pressure $P_{\mathrm{i}}$ will be equal to the fugacity of component $\mathrm{i}$ at $P_{\mathrm{m}}$, the total mixture pressure, after equilibrium has been established. The three general criteria for equilibrium are thus satisfied: the equality of temperature, pressure, and fugacity. The permeation through the membrane, and hence the approach to equilibrium is driven by the equalization of chemical potential on both sides of the membrane.

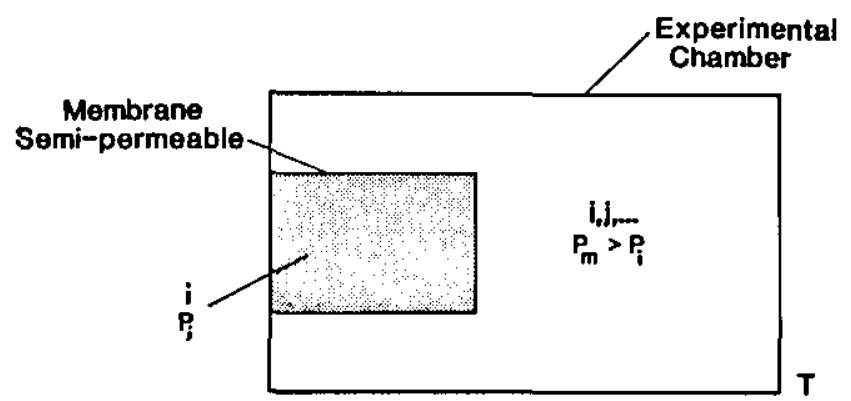

Figure 1-Principle of the semipermeable membrane technique.

The observance and measurement of hydrogen partial pressure was originally done using a semi-permeable membrane by Ramsay [11] and Tsakalotos [12]. The first application of this technique to measurement of gas mixture component fugacities was due to Krishnamurty [13] at Columbia University. His original study of hydrogenhelium mixtures was followed by several studies of hydrogen-propane mixtures $[14,15]$, and a few studies of ternary mixtures containing butane and ammonia with hydrogen-propane $[15,18]$. The present apparatus differs markedly from the original Columbia University instrument, with many of the difficulties $[19,20]$ encountered with the Columbia design being addressed and described in the experimental section.

Once a hydrogen partial pressure has been measured across the semipermeable membrane at a particular temperature, the fugacity of this single component can be determined. It is more convenient to deal with a dimensionless quantity called the fugacity coefficient, $\phi_{i}$, defined by

$$
\phi_{\mathrm{i}}=f_{\mathrm{i}} /\left(x_{\mathrm{i}} P_{\mathrm{i}}\right)
$$

where $P_{\mathrm{i}}$ is the partial pressure of component $\mathrm{i}$ [6]. The fugacity coefficient of this single component can be determined using the virial equation (truncated after the third virial coefficient) $[17,18]$,

$$
\ln \phi_{i}=\frac{B}{R}\left(\frac{P_{\mathrm{i}}}{T}\right)+\frac{C-B^{2}}{2 R^{2}}\left(\frac{P_{\mathrm{i}}^{2}}{T^{2}}\right) .
$$

The above quantities are for pure hydrogen only. To determine the fugacity coefficient of hydrogen in the mixture, we use the defining equation for the fugacity coefficient [10]:

$$
\phi_{\mathrm{H}_{2}}^{m}=f_{\mathrm{H}_{2}} / x_{\mathrm{H}_{2}} P_{\mathrm{m}}
$$

where $x_{\mathrm{H}_{2}}$ is the mole fraction of hydrogen in the mixture, and $P_{\mathrm{m}}$ is the total pressure of the mixture. The value of $P_{\mathrm{m}}$ is necessarily greater than that of $P_{\mathrm{i}}$, since it also includes the partial pressure of the nonpermeating component.

\section{Test Systems}

The systems chosen for the initial studies on this instrument are gaseous binary mixtures of hydrogen/ methane and hydrogen/propane. These were chosen for several reasons. First, there was no evidence that either mixture would be detrimental to the membrane, or that temporary poisoning of the membrane would cause slow diffusion rates. Second, preliminary predictions made using the Redlich-Kwong equation indicated that these systems would be quite informative [8]. Hydrogen/propane is expected to have appreciable nonideality [15], while hydrogen/methane, on the other hand, would be expected to show a smaller change of fugacity coefficient with mole fraction. This second system, therefore, provides a test of the overall sensitivity of the apparatus, since the total expected change is more comparable to the experimental error. Third, there are previous data on hydrogen/propane with which to compare the present data $[18,19]$.

Hydrogen/methane and hydrogen/propane mixtures pose no unfavorable analytical requirements. The chromatographic separations are easily done using one 
column for each mixture. Heart-cutting or multidimensional techniques are unnecessary. The retention times are quite reasonable, and well-shaped peaks are obtained at experimentally convenient column and injector temperatures. The analytical conditions for both mixtures are thus very conducive to precise quantitation.

\section{Experimental}

\subsection{Pressure Vessel}

A schematic diagram of the essential features of the apparatus is given in figure 2. The heart of the apparatus is a large, thick-walled pressure vessel which serves as the experimental chamber. The vessel has a relatively large internal volume $(2050 \mathrm{ml})$, so that the removal of small aliquots of mixture for analysis will cause minimal disturbance of equilibrium. The opening of the vessel is also large (11.43 cm diameter), to accommodate the membrane manifold, a mixer, and required feedthroughs. The vessel was machined from a section of work hardened 316 stainless steel barstock, with final dimensions and clearances exceeding the requirements set forth by the ASME [21,22]. A bolted closure is employed in the vessel, using eight bolts machined from 4340 (AISI designation) steel. The high loading on the

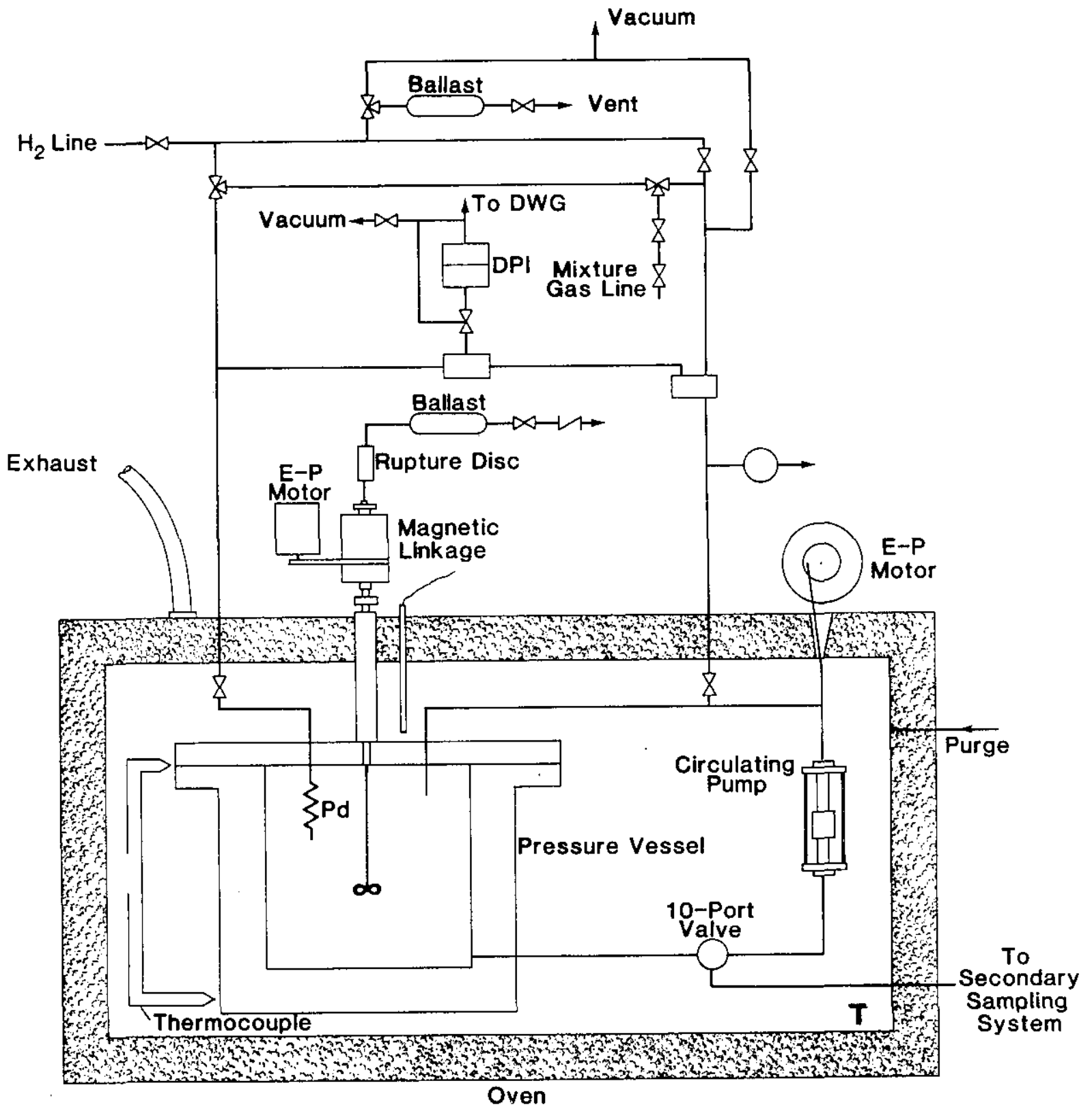

Figure 2-Schematic diagram of the apparatus. 
$11.43 \mathrm{~cm}$ diameter closure required the use of an ultrahigh strength steel for the bolts. Sealing is provided by either a 25\% glass-filled PTFE gasket or 316 stainless steel gasket (the stainless steel gasket is used for temperatures above $220^{\circ} \mathrm{C}$ ). The vessel with its closure is capable of containing a pressure of $50 \mathrm{MPa}$ at $340^{\circ} \mathrm{C}$. The lid of the vessel also accommodates a thermometer probe well, and a provision for a safety head (equipped with a $41 \mathrm{MPa}$ rupture disk) and supports a propellertype mixer. The mixer is a commercial magnetic linkage type with air cooled magnets.

\subsection{Membrane Manifold}

Due to the importance of the membrane manifold for this experiment, and its attendant complexities of fabrication, a detailed discussion is provided. The semipermeable membrane manifold, pictured in figure 3, is suspended from the underside of the vessel lid using a compression fitting.

As stated previously the working principle of the semi-permeable membrane method depends upon the separation of the mixture $(i, j, \ldots)$ and a pure, permeating component (i) into two separate "compartments" within the experimental chamber. The "wall" separating the two components is permeable to $i$ (in the present case, $i$ is hydrogen), but not to the other mixture components. Many materials are known to allow permeation of hydrogen. The best material for this purpose is palladium [23], a fact which has led to commercial exploitation in hydrogen purifiers. Palladium itself has little mechanical strength, however, and in practical applications, one must usually resort to the use of an alloy containing $25 \%$ silver. Palladium-silver, $75 / 25$, is easily fabricated into useful forms such as rod, foil and tubing. Due to geometrical and support considerations, tubing is the most suitable form for use at high pressures.

The Pd-Ag tubing was obtained from a commercial processor and had an outside diameter of $0.160 \mathrm{~cm}$, and a wall thickness of $0.008 \mathrm{~cm}$. The material is annealed to a dead soft condition in a tube furnace, and tested for pinholes using a helium leak detector. At present, the material is available only in $396 \mathrm{~cm}$ long sections, due to limitations of the drawing die. The thin wall of the tubing was necessary to achieve reasonable gas diffusion rates. Indeed, if the wall thickness were much greater, diffusion may not be observed at all [24]. It was necessary to increase the strength of this rather thin and weak tubing, since it must support pressure gradients of $7 \mathrm{MPa}$ or more. For this purpose, $400 \mathrm{~cm}$ length coil springs were fabricated from $0.025 \mathrm{~cm}$ diameter stainless steel wire. The springs were wound to have a nominal relaxed o.d. of $0.145 \mathrm{~cm}$, and are easily inserted into the $\mathrm{Pd}-\mathrm{Ag}$ tubes. The spring is then under a low tension

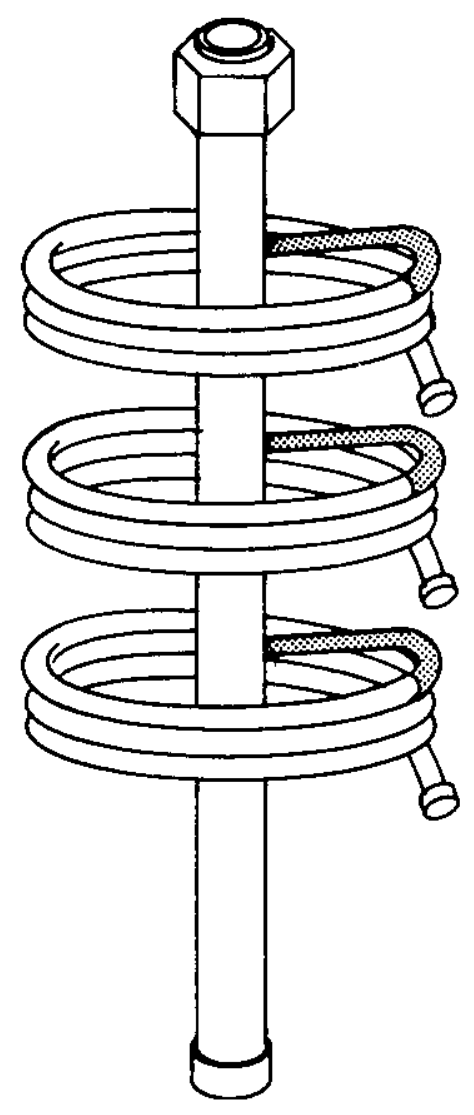

Figure 3-Semipermeable membrane manifold.

interference fit inside the $\mathrm{Pd}-\mathrm{Ag}$ tube, and greatly increases the tube's "compressive load" strength.

The Pd-Ag tubes each containing a coiled spring, were then formed into five layer coils, approximately $2.5 \mathrm{~cm}$ long with a maximum outside coil diameter of 2.5 $\mathrm{cm}$. The coiling process was done on a custom made adjustable length mandrel held in a lathe collet. Extreme care was taken to keep all traces of dirt or oil from contaminating the tubing, since this could result in permanent poisoning of membrane activity [23].

For each manifold (fig. 3), three coils were prepared and wound as described above. One end of each coil (the outside end) was capped off with a custom made 316 stainless steel cap. The other end (the inside end) was fitted into a specially drawn "elbow" made from 0.208 $\mathrm{cm}$ o.d. 321 stainless steel tubing, approximately $1.5 \mathrm{~cm}$ long. This elbow was, in turn, placed into a drilled hole in a central tube $(0.328 \mathrm{~cm}$ o.d., $0.089 \mathrm{~cm}$ wall thickness). The central tube can be made from either 316 stainless steel or 270 nickel. It is the top of this central tube which is ultimately connected to the pressure measurement manifold using a compression fitting. The bottom of the tube is capped.

The Pd-Ag coils, end caps, elbows, and central tube were assembled in a vibration eliminating fixture to al- 
low brazing of all the joints. Due to the chemical sensitivity of Pd-Ag, the use of any kind of flux is impossible. Thus, vacuum brazing was employed. All joints were "dressed" with a single loop of $0.051 \mathrm{~cm}$ diameter precious metal ( $82 \%$ gold, $18 \%$ nickel) brazing wire. The fixture-held manifold was placed in the vacuum furnace, which was then evacuated to $1.3 \times 10^{-5} \mathrm{~Pa}$. The temperature was then raised to the melting point of the brazing wire, and the system was allowed to "soak" at this temperature for a few minutes. The furnace was then cooled slowly to prevent the development of cracks. The completed manifold was tested for pinholes using a helium leak detector, and for hydrogen diffusion (with pure hydrogen) on both sides of the membrane.

Before the membrane is actually used, the Pd-Ag surface must be activated by repeated oxidation and reduction at elevated temperature $\left(300^{\circ} \mathrm{C}\right.$ has proven to be satisfactory). Oxidation is done in a pressurized (6 MPa) environment of oxygen or air; reduction is done in the same way using pure hydrogen [25]. A membrane prepared in this way will quickly reach equilibrium during an experiment. Typically, 45 minutes is adequate to allow $95 \%$ of equilibration (pressure stabilization) to take place.

\subsection{Pressure Measurements}

Measurements of the pressures of the pure hydrogen (from inside the membrane manifold) and the mixture (outside the membrane) were made using a commercial dead weight pressure balance. The balance has an accuracy of $0.015 \%$, and a range of between 0.04 and 82.7 $\mathrm{MPa}$. The pressure of the test fluid (either hydrogen or mixture) is referenced to an inert gas line via a diaphragm type differential pressure transducer. The inert gas communicates its pressure to another differential pressure transducer which is referenced to oil pressure. The oil pressure is generated by weights on a piston table. When both differential pressure transducers are at null condition, one can obtain the pressure of the test fluid by summing the weights pressurizing the piston. Corrections are made for local gravity $(979,601.148$ milligals at Boulder station B), atmospheric pressure, temperature effect on piston area, elastic distortion of the cylinder, and oil head pressure. Corrections for fluid expansion into valve and transfer line volumes are also required. In actual practice, the differential pressure transducers are first brought to null with equal opposing pressures, to allow the setting of a zero reference point. Hysteresis effects in the transducer are minimized by "rocking" the diaphragm up and down several times before taking a measurement. The effect of temperature on the pressure transfer lines (between differential pres. sure transducers) is a small source of systematic error, so care was taken to maintain a uniform room temperature.

\section{Temperature Control and Measurement}

Since the fugacity measurements are made isothermally, adequate temperature control is necessary. Mixed liquid baths are usually the best way to thermostat an experiment of this type. This is impractical in the present work, however, since measurements at high temperatures are anticipated. For this reason, a commercially available vigorously mixed oven was modified to provide a thermostat. The major modifications included the installation of baffles (to promote more uniform mixing of the temperature transmitting fluid), installation of large aluminum thermal masses (to reduce temperature gradients) and the fabrication of a thermally insulated cover plate (from which the pressure vessel and other high temperature components are suspended inside the oven).

The oven is heated by $6.5 \mathrm{~kW}$ resistive elements controlled by a proportional SCR circuit, allowing temperature control of the apparatus components to $\pm 0.05^{\circ} \mathrm{C}$. The controller is also equipped with a high temperature limiting cutoff for safety. For additional safety, the temperature transmitting fluid of the oven is maintained at a slight negative pressure, and a low flow rate of nitrogen is continually passed through the hot zone and is exhausted into a fume hood.

Temperature gradients among key components inside the oven are monitored using opposed pairs of J-type (iron-constantan) thermocouples [26,27]. The observed gradients are then minimized using very low power "shimming" heaters located near or in major components.

Temperature measurements are made using a commercial quartz crystal oscillator thermoprobe. The quartz probe is located in a thermowell in the pressure vessel. The immersion error of the probe in this configuration was calculated to be on the order of $\pm 0.003{ }^{\circ} \mathrm{C}$. A single point calibration is performed on a regular basis, using either the triple point or freezing point of water. This single point measurement is for scaling only; the frequency versus temperature table for the quartz probe is stored on a ROM, and is accessed automatically. The probe has an absolute accuracy of $\pm 0.03{ }^{\circ} \mathrm{C}$ in the present region of interest. Errors due to long term instability amount to less than $\pm 0.008^{\circ} \mathrm{C}$. Errors due to hysteresis are of a negligible level, since the measurements are made isothermally. Thus, thermometry precision on the order of $\pm 0.05 \%$ (coefficient of variation) is easily obtainable.

\section{Analytical System}

It is necessary to determine the composition of the gas mixture (outside the semipermeable membrane) in order 
to deduce fugacity coefficients. A custom designed developmental gas chromatograph and sampling system was built to perform the necessary analyses. This was considered necessary because of some of the unique problems inherent in hazardous gas analysis $[28,29]$.

The analytical system consists of a primary sampling system (which allows removal of a sample from the hostile pressure/temperature environment of the vessel), a secondary sampling system (which allows sample manipulation and injection) and the gas chromatograph, in which the separation and quantitation is actually done. The primary sampling system consists of a 10 port valve (rated for service at $250^{\circ} \mathrm{C}$ and $48 \mathrm{MPa}$ ), a circulating pump, and necessary valving. This equipment is shown in place in figure 2 . The sampling valve is actuated remotely using helium gas (at $0.83 \mathrm{MPa}$, feeding a pneumatic drive), thus permitting the operator to remain outside the potentially hazardous pressure vessel zone. Helium is used as the actuation fluid to provide very fast valve switching. The evacuable sample loop of this valve has a volume of $0.5 \mathrm{ml}$. At a mixture pressure of $3.45 \mathrm{MPa}$, the withdrawal of a sample aliquot of this size causes a mixture pressure drop of $0.0008 \mathrm{MPa}$, which has a negligible effect upon the equilibrium mixture composition. Before the sampling valve is switched to remove this plug of sample, the mixture gas is circu- lated through the sample loop using a commercial check valve type pump. This minimizes the problem of concentration gradients, which often plague expansions of gas mixtures from elevated pressures. Only a few minutes of circulation are needed, since the pump is able to move $4.0 \mathrm{ml}$ of fluid per stroke, and a pumping rate of 15 strokes per minute is employed.

Switching of the primary sampling valve results in the transfer of the plug of sample to the secondary sampling manifold, which is shown schematically in figure 4 . The sample is held between valves A and B inside a $316 \mathrm{~L}$ stainless steel bellows. The pressure of the sample may be varied by compressing or expanding the bellows. Valve $B$ allows the sample to be introduced into a 10 port sampling valve, S, equipped with a $50 \mu 1$ sample loop. Valve $\mathrm{D}$ allows the loop to be evacuated before being filled with sample and between analyses. It has been found in this work that loop evacuation before filling leads to a factor of two increase in precision of the measured chromatographic peak area counts. The vent valve, $\mathrm{C}$, allows the sample pressure to be dropped to ambient before injection. The ambient pressure is measured using a fixed cistern mercury barometer, with the appropriate corrections being applied to the readings [30].

The sampling valve, bellows, and valves A through D
Figure 4-Secondary sampling manifold.

To Chromatograph
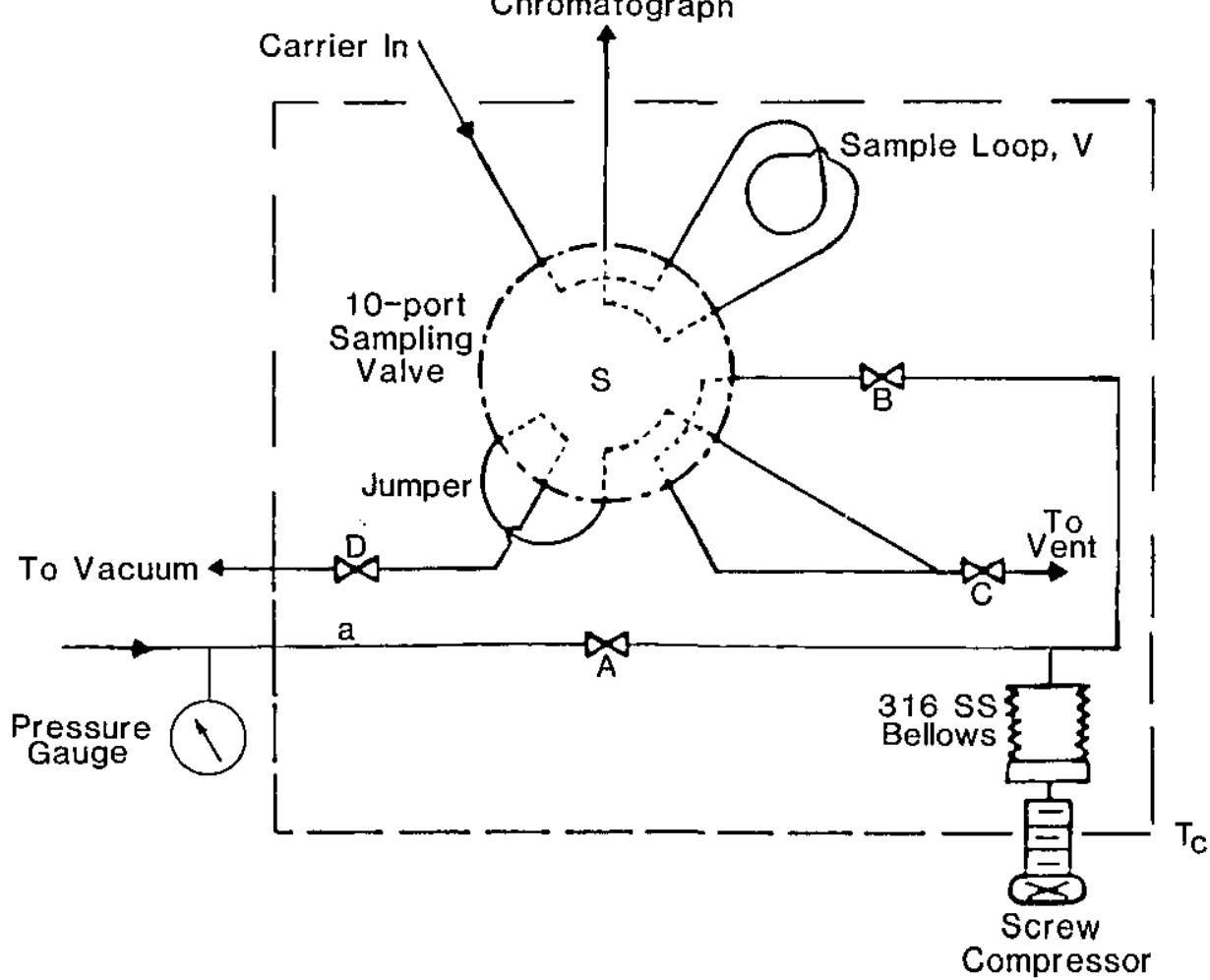
are mounted within a massive $\left(1050 \mathrm{~cm}^{3}\right)$ aluminum block which is heated (in the present work, to $125 \pm 0.3^{\circ} \mathrm{C}$ ). The aluminum block reduces temperature gradients to a negligible level. Temperature control is provided by a proportional controller which responds to a thermistor sensor. Thus, by controlling the temperature and pressure of the fixed volume sample loop, one is assured of injecting the same quantity of sample for each analysis.

Upon switching the sampling valve of the secondary manifold, the plug of sample is swept from the loop into the chromatograph. This chromatograph has been specially constructed to provide precise control of column temperature and pressure. The column is thermostated in a vigorously stirred oil bath which is maintained to within $\pm 0.02^{\circ} \mathrm{C}$. All analyses are done isothermally to minimize carrier flow rate disruptions. Column pressure is controlled using a fine adjustment pressure regulator (a commercially available unit used for pressure gauge calibration) followed by a fine metering valve. The chromatograph is very similar to units used for precise thermodynamic measurements [31-33]. Mass flow controllers are not used since the column temperature is not programmed. The carrier gas flow rate, measured at the detector exit, is maintained at $40.00 \pm 0.05 \mathrm{ml}$ per minute. Since both hydrogen and the mixture gas (in the present case, methane and propane were studied) required detection and quantitation, argon was chosen as the carrier gas [34].

The separation of mixtures of hydrogen-methane and hydrogen-propane is experimentally trivial and will be described later. A micro cell thermal conductivity detector (TCD) at the column exit, connected to a commercial electronic integrator, provided detection and quantitation. Peak area counts as logged by the integrator are corrected for atmospheric pressure (equivalent to sample loop pressure, since injections were done at ambient pressure) and detector aging. The pressure correction is required since the loop pressure affects the quantity of fluid injected, and dividing by the atmospheric pressure normalizes the response. The correction for detector aging is needed since the goldsheathed tungsten filaments of the TCD run hotter in an argon carrier stream than in the more common carrier gases such as helium and hydrogen. Thus, the filaments undergo accelerated oxidation due to trace amounts of oxygen that are not trapped. This correction is applied by periodically injecting a standard mixture to track signal loss. The corrected peak areas are then converted to mole fractions either by using a calibration equation or the standard bracketing technique $[35,36]$. After leaving the detector exit, sample and carrier are expelled through a transfer line to a dedicated outside blower, to provide operator safety.

\section{Testing and Evaluation}

Since the analytical measurements are the greatest single source of error in this experiment, a thorough testing of the sampling and chromatographic systems was needed. Testing of the nonanalytical components of the apparatus was described in the preceding section. In this section, the extensive tests performed on the analytical system will be described.

Due to the complexity of the two sampling systems, with many joints, fittings and valves, correlation between repeated measurements is very possible. For example, if sampling valves are not sufficiently evacuated or purged, residual sample from previous analyses would cause a systematic, additive error. This would be reflected in a definite trend of component areas with analysis repetition or time.

To check for this type of sample correlation, an extended study was done on a standard mixture of $74.8 \%$ hydrogen, $25.2 \%$ methane. The mixture was prepared as described (for standard calibration mixtures) in the next section. Thirty separate analyses were done on the mixture over a period of two days. The measurements were made in approximately equal intervals of time. Component areas, total areas, and component chromatographic mole fractions were calculated. Chromatographic mole fractions are those determined from the component area and total area, uncorrected for relative detector response. It was then necessary to look for trends in these measured quantities as a function of sample run or time.

Plots of each of the above quantities versus run (or time) revealed no discernible structure. In addition, tables of correlation were calculated, which included Pearson, Spearman, and Kendall correlation coefficients. No unexpected correlations were observed. Related variables, such as component area and total area, showed high correlation as expected $[37,38]$. Linear regressions were performed in which the measured chromatographic quantities were fit against the repetition number. In each case, little correlation was found. The estimates of the first order parameter were poorly determined (as deduced from the standard errors) and of questionable significance (as deduced from the $t$ values), while the intercepts were well determined and highly significant. This suggests that the proper representation for repeat analysis data is a mean with some measure of dispersion. Since the data were taken at approximately equal intervals of time, time, series statistics are nominally informative. Thus, first order auto-correlation and Durbin-Watson $d$ statistics were calculated, and indicated minimal correlation among errors [39]. Since an average appears to represent repetitive analysis adequately, we can make probability plots to assess the 
nature of the distribution of data about the mean. These tests showed that the actual distribution of measurement values deviates negligibly from the normal distribution, and inferences based on the assumption of normality are valid [40].

It may be concluded from the results of foregoing tests that each analysis is an independent measurement of concentration, unaffected by previous measurements, and that an average of repetition measurements will provide an unbiased estimate of concentration, and the standard deviation will provide a measure of the dispersion.

\section{Quantitation}

Since the sensitivity of the thermal conductivity detector (TCD) is sample material dependent, it is not possible to take the integrator response as a concentration reading. It should be noted that absolute detectors requiring no calibration are available (for example, a gas density balance), and for these instruments direct integrator response is a valid method of quantitation. For relative response detectors such as the TCD, one must use either an internal or external standard, or normalization $[35,36]$. For this type of work, the external standard method is preferable.

To use the external standard method, mixtures of known composition of hydrogen/methane and hydrogen/propane were prepared. These standard gas mixtures were then chromatographed under the same conditions as the experimental mixtures (same conditions of carrier flow rate, injector, column and detector temperature, and TCD bridge current). The standard mixtures were prepared gravimetrically using a commercial $25 \mathrm{~kg}$ capacity two pan balance. The sensitivity of this balance is $\pm 0.0025 \mathrm{~g}$, with an accuracy dependent on calibration with a standard $1 \mathrm{~g}$ class $\mathrm{S}$ weight $(1.0000 \pm 0.0005 \mathrm{~g})$ [41]. This uncertainty will propagate an error of approximately $0.4 \%$ into a typical gravimetrical mole fraction (a mixture of 0.5 mole fraction hydrogen prepared by three separate weight measurements).

\section{Hydrogen-Methane Calibration and Analysis}

Nine standard mixtures of hydrogen-methane were prepared for the calibration, along with a sample of pure hydrogen. Pure methane was not used since it represents an experimentally unaccessible point in the mole fraction range. Separation was easily done using a packed column ( $1.5 \mathrm{~m}$ long, $0.32 \mathrm{~cm}$ o.d.) of $150-200$ mesh $5 \AA$ molecular sieve. The column was maintained at $60^{\circ} \mathrm{C}$, since methane tends to adsorb on the zeolite surface of the molecular sieve at lower temperatures. The elevated temperature also provided for increased speed of analysis without sacrificing baseline resolution of the peaks. A plot of the corrected area counts (detector response) versus mole fraction is provided in figure 5 . The slight but noticeable curvature of this plot is not unusual for

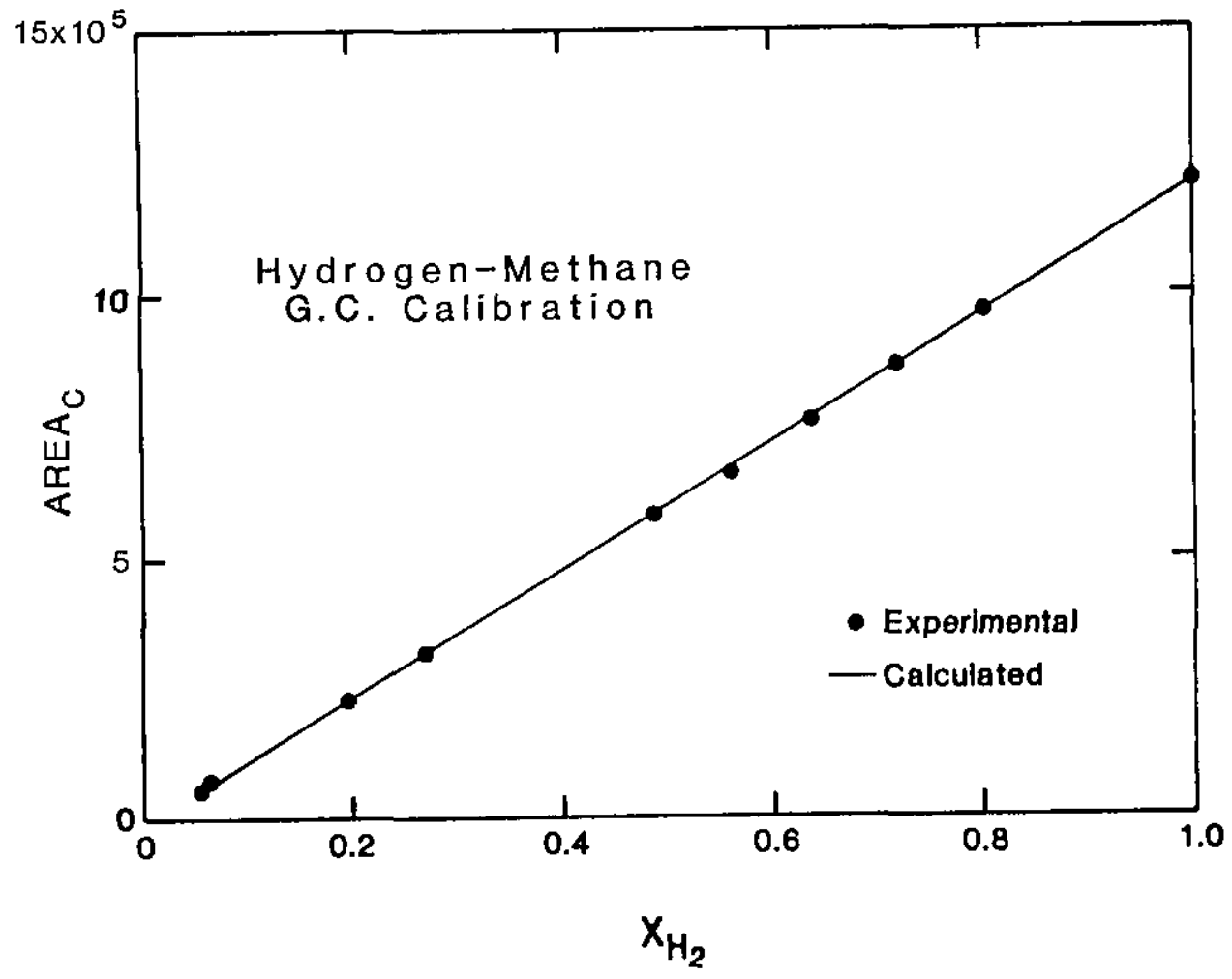

Figure 5-Chromatographic calibration curve for hydrogenmethane binary. 
hydrogen-containing binary mixtures.

The data were fitted to a second degree polynomial, with the result that the poorly determined constant term proved to be statistically indistinguishable from zero. This is internally consistent, since the curve must logically pass through the origin. A second degree polynomial constrained to pass through the origin (i.e., the constant term is set equal to zero) was then found to represent the data to within experimental error. The results of the analysis are shown in table 1 . The resulting parameters $a$ and $b$ were highly significant (as judged by the $t$-test and the $F$-test), although $a$ is far better determined than is $b$. The percent error in the fit is 0.55 , which is comparable to that obtained using other chromatographic quantitation methods. The root mean square error, or overall regression standard deviation, was \pm 0.0026 in mole fraction, resulting in an error of $0.52 \%$ in mole fraction for an equimolar mixture. A plot of residuals versus mole fraction showed no discernible structure, and a regression found no meaningful correlation. Thus, the model set forth in table 1 accounts for all data structure except random error.

Table 1. Regression results for hydrogen-methane calibration

$$
a A+b A^{2}=n
$$

where $\quad A=$ corrected chromatographic area response $n=$ mole fraction of hydrogen

regression results:

$$
\begin{aligned}
a & =8.5764 \times 10^{-7} \quad 0.56 \text { percent } \mathrm{CV} \\
b & =-2.5940 \times 10^{-14} 38.70 \text { percent } \mathrm{CV} \\
r^{2} & =0.99998
\end{aligned}
$$

root mean square error, $n=0.0026$

coefficient of variation $=0.5506$

\section{Hydrogen Propane Calibration and Analysis}

Standard calibration mixtures were prepared for hydrogen-propane in the same way as for hydrogenmethane. For this system, only four standard mixtures were prepared, and only in the higher hydrogen mole fraction range. Calculations were done using the standard "bracketing" method [29] rather than by determining a calibration equation. The reason for this type of calibration (rather than the more preferable type done for hydrogen-methane) is the very high cost of research grade propane. It is, however, not unreasonable to expect mole fraction accuracy of better than $1.0 \%$ for the hydrogen-propane system.
This mixture is easily separated using a packed column ( $1.5 \mathrm{~m}$ long, $0.32 \mathrm{~cm}$ o.d.) containing porapak- $\mathrm{Q}^{2}$. The column was maintained at $80^{\circ} \mathrm{C}$, since the porous polymer packing was observed to entrain hydrogen at lower temperatures. It should be noted that elevated temperatures cause the polymer beads to swell, resulting in an increase in the retention time of hydrogen and a decrease of that of propane. Thus, the temperature at which the two peaks merge is lowered by these additive effects.

\section{Results for Hydrogen/Propane and Hydrogen/Methane}

Plots of the fugacity coefficient of hydrogen in mixture with propane, $\phi_{\mathrm{H}_{2}}^{m}$, as deduced from eqs (2), (3), and (4), are shown in figures 6 and 7. The data shown in figure 6 were taken at $80^{\circ} \mathrm{C}(353 \mathrm{~K})$, and those in figure 7 were taken at $130^{\circ} \mathrm{C}(403 \mathrm{~K})$. The nominal mixture (total) pressure in each case was $3.45 \mathrm{MPa}$. The actual data are listed in tables 2 and 3. The error bars representthe uncertainty of a typical $\phi_{\mathrm{H}_{2}}^{\mathrm{m}}-x_{\mathrm{H}_{2}}$ pair, and will be discussed in more detail later. The data represented by the triangles on figure 6 are the results of an earlier study [17]. Although the earlier data were taken at a slightly different temperature $(345 \mathrm{~K}$ instead of the $353 \mathrm{~K}$ in the present work), the agreement is striking.

In both plots, the change in $\phi_{\mathrm{H}_{2}}^{m}$ is most pronounced at lower values of $x_{\mathrm{H}_{2}}$. This behavior is consistent for a low molecular weight gas (at a relatively high reduced temperature) in a binary mixture with a heavier gas. It should also be noted that the data at $130^{\circ} \mathrm{C}$ show a more gentle increase in slope than the data at $80^{\circ} \mathrm{C}$. This is consistent with the expectation that $\phi_{\mathrm{H}_{2}}^{m}$ be closer to unity at higher temperatures, for a given composition. The slight upturn at the high hydrogen mole fraction range is probably due to the upturn in molar volume in this region. This behavior of $\phi_{\mathrm{H}_{2}}^{m}$ is also suggested in an earlier study done under approximately the same conditions [15]. A study comparing the measured values of $\phi_{\mathrm{H}_{2}}^{m}$ with predictions of several equations of state is ongoing and will be reported later [42].

A plot of $\phi_{\mathrm{H}_{2}}^{m}$ for hydrogen/methane at $80^{\circ} \mathrm{C}(353 \mathrm{~K})$ and $3.45 \mathrm{MPa}$ is provided in figure 8 . The actual data are listed in table 4 . The total change of $\phi_{\mathrm{H}_{2}}^{m}$ over the hydrogen mole fraction range studied is $13 \%$, compared to

\footnotetext{
${ }^{2}$ Certain commercial equipment, instruments, or materials are identified in this paper in order to adequately specify the experimental procedure. Such identification does not imply recommendation or endorsement by the National Bureau of Standards, nor does it imply that the materials or equipment identified are necessarily the best available for the purpose.
} 
Figure 6-Fugacity coefficient of hydrogen, $\phi_{\mathrm{H}_{2}}^{m}$, versus mole fraction of hydrogen, in propane at $80^{\circ} \mathrm{C}(353 \mathrm{~K})$. A typical error bar is shown at the lower left.

0 - this work

$\Delta$ - previous data of Antezana [17], taken at $345 \mathrm{~K}$.
Figure 7-Fugacity coefficient of hydrogen, $\phi_{\mathrm{H}_{2}}^{m}$, versus mole fraction of hydrogen, in propane at $130{ }^{\circ} \mathrm{C}(403 \mathrm{~K})$. A typical error bar is shown at upper right.
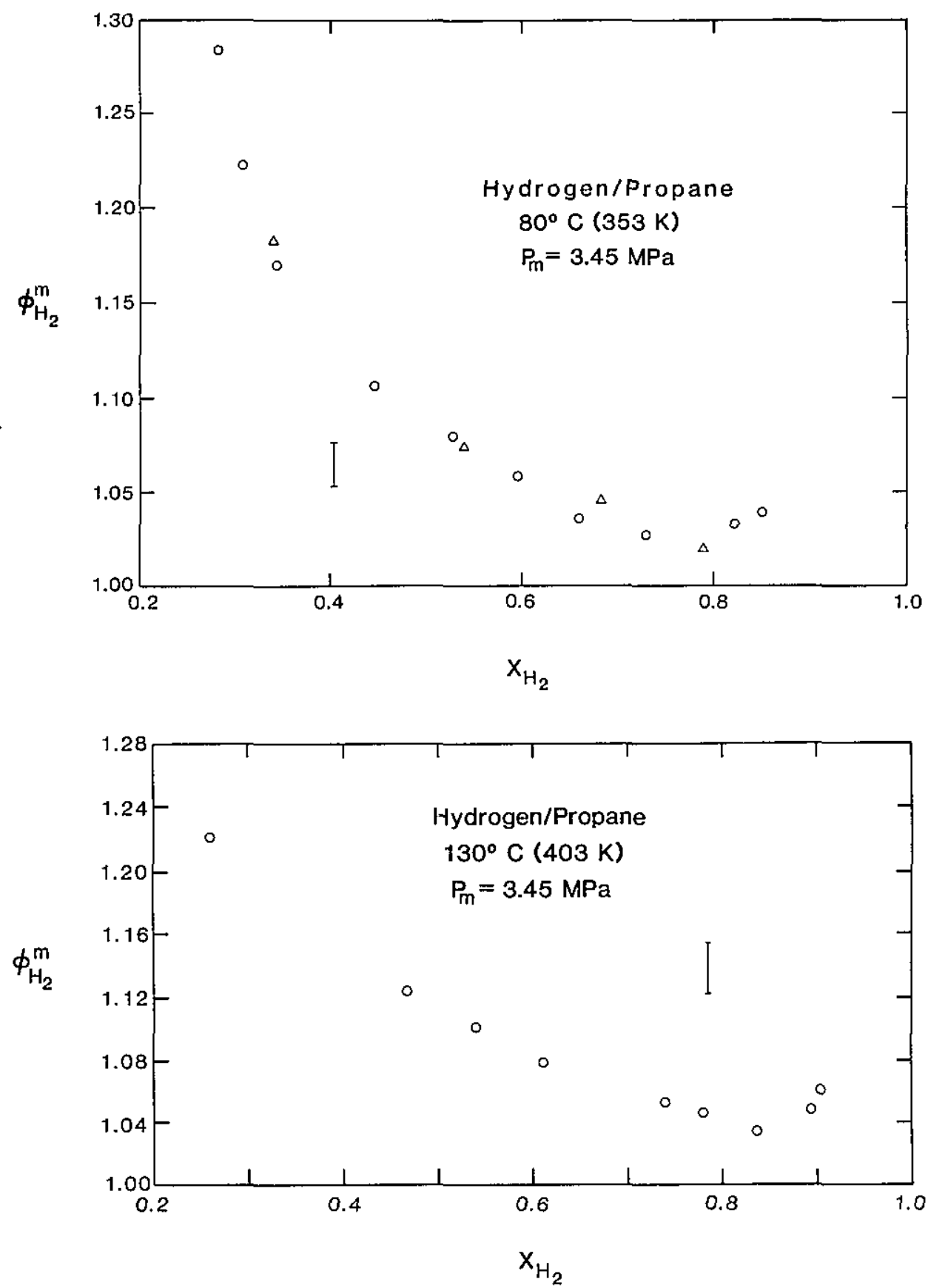

$23 \%$ in the hydrogen-propane mixture at $353 \mathrm{~K}$. This is expected because of the higher reduced temperature of methane. The value of $\phi_{\mathrm{H}_{2}}^{m}$ changes most sharply in the region of low hydrogen mole fraction, as expected [10]. Data on this system were not taken above $353 \mathrm{~K}$ since the change in $\phi_{\mathrm{H}_{2}}^{m}$ would probably not be detected. As with the hydrogen-propane system, work on predictions and comparison with equation of state results is in progress and will be reported on later.

The error bars shown in figures 6-8 represent random propagated errors in $\phi_{\mathrm{H}_{2}}^{m}$ and $x_{\mathrm{H}_{2}}$. From eq (2), only the first term on the righthand side was considered, since the contribution of the second order term is small. Errors in temperature, hydrogen partial pressure, and the second virial coefficient were then considered. Strictly, the error in B might properly be considered systematic, however enough data are available on hydrogen to ascribe a measure of dispersion about $B$ [43-45]. The error propagation for eqs (3) and (4) was carried out in the usual manner [46]. The recognized sources of systematic error, along with countermeasures, have been described in the experimental section. 
Table 2. Hydrogen/propane at $80^{\circ} \mathrm{C}(353 \mathrm{~K})$

Mole fraction, $\mathrm{H}_{2} \quad$ Partial pressure, $\mathrm{H}_{2} \quad$ Fugacity coefficient, $\phi_{\mathrm{H}_{2}}^{m}$ (MPa)

\begin{tabular}{lll}
\hline 0.848 & 3.01 & 1.040 \\
0.822 & 2.91 & 1.033 \\
0.730 & 2.73 & 1.028 \\
0.659 & 2.33 & 1.036 \\
0.594 & 2.15 & 1.058 \\
0.526 & 1.96 & 1.080 \\
0.445 & 1.69 & 1.106 \\
0.344 & 1.39 & 1.170 \\
0.309 & 1.33 & 1.230 \\
0.280 & 1.25 & 1.283 \\
\hline
\end{tabular}

Table 3. Hydrogen/propane at $130^{\circ} \mathrm{C}(403 \mathrm{~K})$

Mole fraction, $\mathrm{H}_{2} \quad$ Partial pressure, $\mathrm{H}_{2} \quad$ Fugacity coefficient, $\phi_{\mathrm{H}_{2}}$ (MPa)

\begin{tabular}{lll}
\hline 0.904 & 3.29 & 1.058 \\
0.897 & 3.23 & 1.054 \\
0.835 & 2.96 & 1.038 \\
0.783 & 2.81 & 1.047 \\
0.738 & 2.66 & 1.047 \\
0.618 & 2.28 & 1.077 \\
0.545 & 2.06 & 1.096 \\
0.472 & 1.82 & 1.116 \\
0.265 & 1.12 & 1.220 \\
\hline
\end{tabular}

Table 4. Hydrogen/methane at $80^{\circ} \mathrm{C}(353 \mathrm{~K})$

\begin{tabular}{ccc}
\hline \hline Mole fraction, $\mathrm{H}_{2}$ & $\begin{array}{c}\text { Partial pressure, } \mathrm{H}_{2} \\
(\mathrm{MPa})\end{array}$ & Fugacity coefficient, $\phi_{\mathrm{H}_{2}}$ \\
\hline 0.849 & 3.17 & 1.121 \\
0.790 & 2.92 & 1.115 \\
0.764 & 2.80 & 1.097 \\
0.652 & 2.40 & 1.115 \\
0.536 & 1.99 & 1.125 \\
0.459 & 1.75 & 1.134 \\
0.307 & 1.19 & 1.166 \\
0.216 & 0.88 & 1.223 \\
\hline
\end{tabular}

The author would like to thank Drs. Gerald C. Straty and Howard J. M. Hanley for many helpful discussions, and Gretchen L. Hume for her assistance in performing some of the measurements.

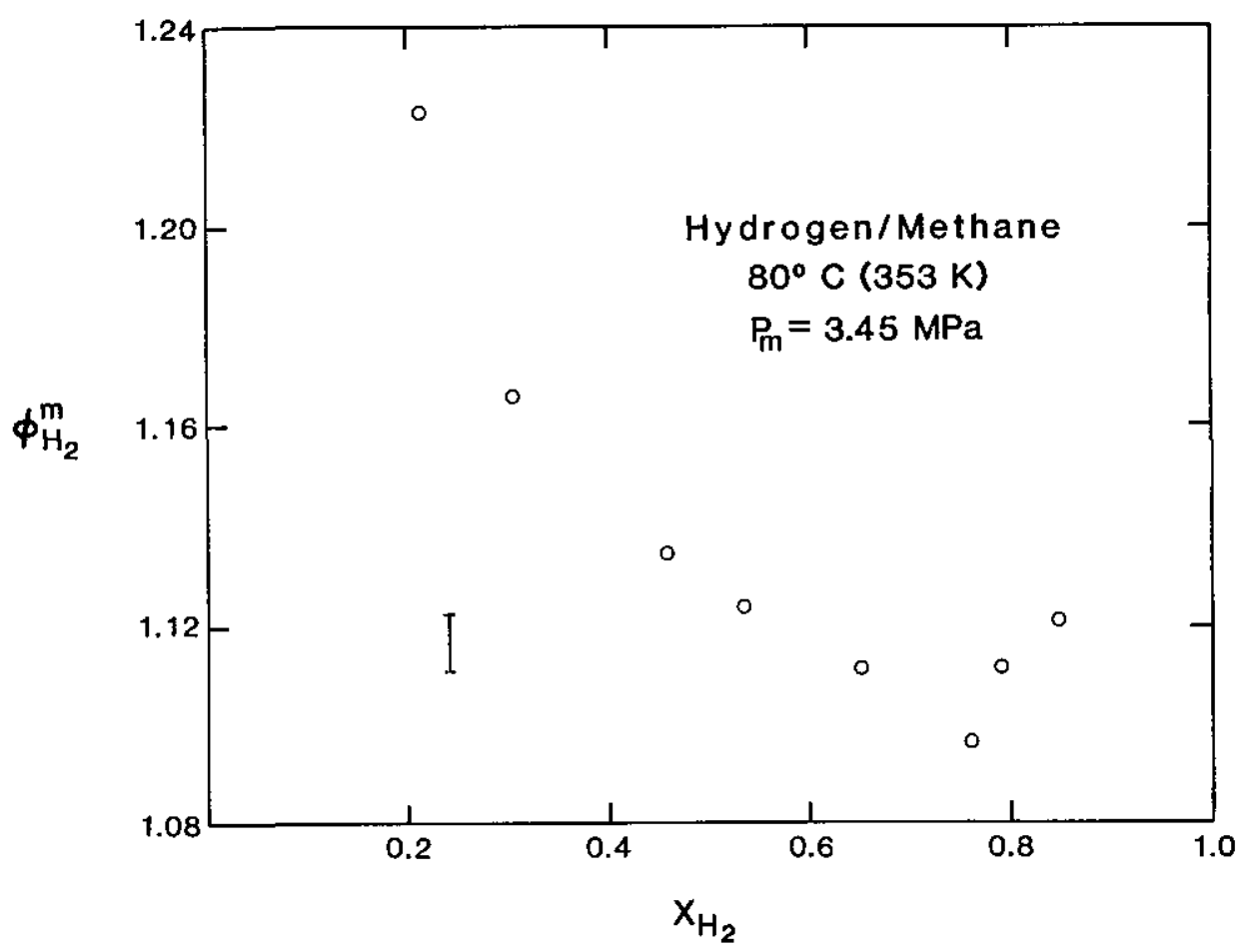

Figure 8-Fugacity coefficient of hydrogen $\phi_{\mathrm{H}_{2}}^{m}$, versus mole fraction of hydrogen, in methane at $80^{\circ} \mathrm{C}(353 \mathrm{~K})$. A typical error bar is shown at the lower left. 


\section{References}

[1] Lewis, G. N., Proc. Am. Acad. 37, 49 (1901).

[2] Lewis, G. N., Z. Physik. Chem. 38, 205 (1901).

[3] Lewis, G. N., and M. Randall, Thermodynamics. New York: McGraw-Hill Book Co. (1961-revised).

[4] Sandler, S. I., Chemical and Engineering Thermodynamics. New York: John Wiley and Sons (1977).

[5] Kyle, B. G., Chemical and Process Thermodynamics. Englewood Cliffs: Prentice Hall, Inc. (1984).

[6] Prausnitz, J. M., Molecular Thermodynamics of Fluid-Phase Equilibria. Englewood Cliffs: Prentice Hall, Inc. (1969).

[7] Prausnitz, J. M., A.I.Ch.E. J. 5, 3 (1959).

[8] Chao, K. C., and R. A. Greenkorn, Thermodynamics of Fluids. New York: Marcel Dekker, Inc. (1975).

[9] Denbigh, K., The Principles of Chemical Equilibrium. Cambridge University Press (1971 3rd Ed.).

[10] Cheh, H. Y., Proceedings of the 6th symposium on thermophysical properties. ASME, 256 (1973).

[11] Ramsay, W., Phil. Mag. 38(5), 206 (1894).

[12] Tsakalotos, D., Proc. Chem. Soc. London 24, 208 (1908).

[13] Krishnamurty, V. V. G., doctoral dissertation. New York: Columbia University (1963).

[14] Cooper, H. W., doctoral dissertation. New York: Columbia University (1967).

[15] Ghosal, A. B., doctoral dissertation. New York: Columbia University (1971).

[16] Klink, A. E.; H. Y. Cheh and E. H. Amick, A.I.Ch.E. J. 21, 1142 (1975).

[17] Antezana, F., and H. Y. Cheh, Ind. Eng. Chem. Fundam. 14, 224 (1975).

[18] Antezana, F., and H. Y. Cheh, Ind. Eng. Chem. Fundam. 15, 95 (1976).

[19] Klink, A. E., private communication (1981).

[20] Antezana, F., private communication (1981).

[21] ASME boiler and pressure vessel code, Sec. VIII: Unfired pressure vessels. New York: American Society of Mechanical Engineers (1965).

[22] Oberg, E., and F. D. Jones, Machinery's Handbook, H. L. Horton, ed. New York: The Industrial Press $(1964,2104$ p.).
[23] Smith, D. P., Hydrogen in Metals. Chicago: University of Chicago Press (1948)

[24] Wise, E. M., Palladium. New York: Academic Press (1968).

[25] McGee, W., private communication (1981).

[26] Sparks, L. L., private communication (1982).

[27] Powell, R. L.; W. J. Hall, C. H. Hyink, L. L. Sparks, G. W. Burns, M. G. Scroger, and H. H. Plumb, Thermocouple reference tables based on the IPTS-68. Natl. Bur. Stand. (U.S.) Monogr. 125 (1974).

[28] Jeffery, P. G., and P. J. Kipping, Gas Analysis by Gas Chromatography. Oxford: Pergamon Press (1972).

[29] Cowper, C. J., and A. J. DeRose, The Analysis of Gases by Chromatography. Oxford: Pergamon Press (1983).

[30] Brombacher, W. G.; D. P. Johnson, and J. L. Cross, Mercury barometers and manometers. Natl. Bur. Stand. (U.S.) Monogr. 8 (1964).

[31] Conder, J., and R. Young, Physicochemical Measurement by Gas Chromatography. Chichester: John Wiley and Sons (1979).

[32] Laub, R. J., and R. L. Pecsok, Physicochemical Applications of Gas Chromatography. New York: Wiley Interscience (1978).

[33] Bruno, T. J.; D. E. Martire, M. W. P. Harbison, A. Nikolic, and C. F. Hammer, J. Phys. Chem. 87, 2425 (1983).

[34] Heftmann, E., Chromatography; A Laboratory Handbook of Chromatographic and Electrophoretic Methods. New York: Van Nostrand Reinhold (1975, 3rd Ed.).

[35] McNair, H. M. and E. J. Bonnelli, Basic Gas Chromatography. Varian Aerograph (1969).

[36] Grant, D. W., Gas Liquid Chromatography. London: Van Nostrand Reinhold (1971).

[37] S.A.S. Users Guide: Statistics. Cary, NC: SAS Institute (1982).

[38] Langley, R., Practical Statistics. New York: Dover (1970).

[39] Ostle, B., and R. W. Mensing, Statistics in Research. Ames, IA: Iowa State University Press (1975).

[40] Vecchia, D. L., private communication (1984).

[41] Battino, R., and A. G. Williamson, J. Chem. Ed. 61, 51 (1984).

[42] Ely, J. F., to be published.

[43] Levelt-Sengers, J. M. H.; M. Klein, and J. S. Gallagher, AEDCTR-71-39; USAF (1971)

[44] Goodwin, R. D., private communication (1984).

[45] McCarty, R. D., private communication (1984).

[46] Meyer, S. L., Dato Analysis for Scientists and Engineers. New York: John Wiley and Sons (1975). 